Nouveaux cahiers de la recherche en éducation

\title{
Évolution de l'engagement parental auprès des élèves en difficulté de comportement et des élèves ordinaires au début du secondaire
}

\author{
Julie Marcotte, Laurier Fortin, Richard Cloutier, Égide Royer et Diane Marcotte
}

Volume 8, numéro 2, 2005

Groupe de recherche sur les inadaptations sociales de l'enfance

URI : https://id.erudit.org/iderudit/1017528ar

DOI : https://doi.org/10.7202/1017528ar

Aller au sommaire du numéro

Éditeur(s)

Faculté d'éducation, Université de Sherbrooke

ISSN

1911-8805 (numérique)

Découvrir la revue

Citer cet article

Marcotte, J., Fortin, L., Cloutier, R., Royer, É. \& Marcotte, D. (2005). Évolution de l'engagement parental auprès des élèves en difficulté de comportement et des élèves ordinaires au début du secondaire. Nouveaux cahiers de la recherche en éducation, 8(2), 47-56. https://doi.org/10.7202/1017528ar
Résumé de l'article

L'objectif de cette étude est d'évaluer l'évolution de l'engagement parental au cours des trois premières années du secondaire chez les garçons et les filles appartenant à deux groupes distincts : élèves en difficulté de comportement ou élèves ordinaires. À partir d'un échantillon de 300 élèves (150 ordinaires et 150 en difficulté), les analyses effectuées suggèrent un profil d'engagement parental à la baisse pour les élèves des deux groupes. Or, des analyses effectuées en fonction du genre de l'élève permettent d'identifier un profil d'engagement différent pour le sous-groupe des filles en difficulté de comportement; ce profil d'engagement parental ne serait pas caractérisé par une baisse aussi généralisée comparativement à celui identifié pour les autres élèves. 


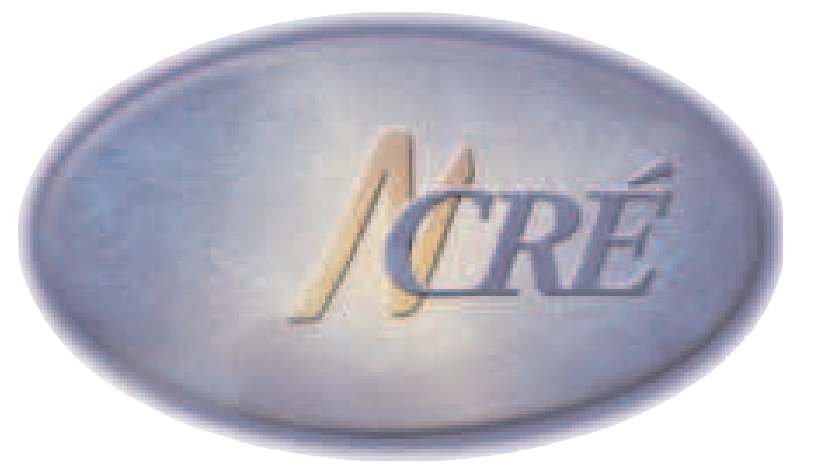

\title{
Évolution de
}

\section{l'engagement parental} auprès des élèves en difficulté de comportement et des élèves ordinaires au début du secondaire

\author{
Julie Marcotte et Laurier Fortin \\ Université de Sherbrooke \\ Richard Cloutier et Égide Royer \\ Université Laval \\ Diane Marcotte \\ Université du Québec à Montréal
}

Résumé - L'objectif de cette étude est d'évaluer l'évolution de l'engagement parental au cours des trois premières années du secondaire chez les garçons et les filles appartenant à deux groupes distincts : élèves en difficulté de comportement ou élèves ordinaires. À partir d'un échantillon de 300 élèves (150 ordinaires et 150 en difficulté), les analyses effectuées suggèrent un profil d'engagement parental à la baisse pour les élèves des deux groupes. Or, des analyses effectuées en fonction du genre de l'élève permettent d'identifier un profil d'engagement différent pour le sous-groupe des filles en difficulté de comportement; ce profil d'engagement parental ne serait pas caractérisé par une baisse aussi généralisée comparativement à celui identifié pour les autres élèves.

Abstract - The goal of this study was to evaluate the progress of parental involvement during the first three years of secondary school for boys and girls belonging to two distinct groups, pupils with behavioural difficulties and ordinary pupils. Based on a sample of 300 pupils (150 ordinary ones and 150 pupils with difficulties), our analyses suggest a profile of decreasing parental involvement for both groups. Analyses by pupils' sex revealed a different parental involvement profile for the sub-group of girls with behavioural difficulties : it is not characterized by as broadly generalized a decrease, in comparison with that observed for other pupils. 


\section{Problématique}

La famille, la source de socialisation la plus précoce et la plus fondamentale pour l'enfant en développement, continue de jouer un rôle déterminant à l'adolescence malgré les transformations qui se bousculent au cours de cette période (Tap et Oubrayrie, 2002). Ainsi, les parents, qui sont en mesure de s'ajuster aux besoins changeants de leurs adolescents, notamment en ce qui a trait à une autonomie grandissante, faciliteraient l'emprunt de trajectoires personnelles et éducationnelles positives (Wallis et Barrett, 1998). Les processus familiaux tels le style qui caractérise le parent dans sa façon d'intervenir auprès du jeune, son implication dans le suivi scolaire ou les règlements imposés, sont des transactions quotidiennes qui dévoilent le degré d'engagement de la famille. D'ailleurs, ces transactions auraient davantage d'importance dans l'ajustement scolaire du jeune que la structure de sa famille et les ressources dont elle dispose (Bogenschneider, 1997; Deslandes, 1996). Des études récentes montrent que le degré d'engagement des parents et leur façon d'interagir avec leur adolescent varient selon que le jeune connaît ou non des difficultés en contexte scolaire et en fonction du genre (Deslandes, Leclerc et DoréCôté, 2001; Marcotte, 2004) en plus de se modifier avec l'âge de l'adolescent (Marcotte, 2004). Le but de cette étude est d'abord d'identifier l'évolution du degré d'engagement parental auprès des élèves suivant un parcours ordinaire et des élèves en difficulté au cours des trois premières années au secondaire et, dans un deuxième temps, de connaître l'effet du genre sur la variation de ces profils.

\subsection{L'engagement parental: le style et les pratiques parentales}

Le style parental représente une constellation d'attitudes et de pratiques qui sont communiquées à l'enfant et à l'adolescent. Les adolescents exposés à un style parental démocratique, caractérisé par des niveaux élevés de contrôle et de sensibilité, sont perçus comme étant plus compétents au point de vue social et scolaire en plus de posséder une meilleure estime d'euxmêmes, davantage de jugement moral et d'autocontrôle (Steinberg, 2000). Trois composantes inhérentes au style parental démocratique favorisent l'ajustement scolaire des adolescents: un niveau élevé d'engagement parental, un degré élevé d'encadrement parental et un degré élevé d'encouragement à l'autonomie (Darling et Steinberg, 1993; Steinberg, 2000).

En plus du style éducatif parental, l'ajustement du jeune en milieu scolaire risque d'être affecté par des pratiques parentales plus spécifiquement liées à l'interface école-famille (Holmbeck, Paikoff et Brooks-Gunn, 1998). Aussi, plusieurs auteurs se sont intéressés aux pratiques parentales de suivi scolaire qui reflètent, plus spécifiquement, l'engagement parental dans la scolarisation du jeune (Epstein, 1992).

Les pratiques parentales de suivi scolaire renvoient aux rôles et à l'accompagnement des parents dans le cheminement scolaire de leurs jeunes. Inspirée des travaux d'Epstein (1992), Deslandes (1996) estime que ces pratiques spécifiques peuvent être regroupées en cinq dimensions: 1) le soutien affectif, 2) la communication avec les enseignants, 3) les interactions parents-adolescent relatives au quotidien scolaire, 4) la communication parents-école, et 5) la communication parents-adolescent. Au secondaire, les résultats les plus probants montrent que les adolescents, dont les parents participent activement au suivi scolaire, ont un meilleur rendement, de plus grandes aspirations scolaires, manifestent moins de difficultés scolaires et 
abandonnent moins souvent l'école avant l'obtention du diplôme d'études secondaires (Deslandes, 1996; Steinberg, Lamborn, Dornbusch et Darling, 1992). L'engagement parental, tel que conceptualisé à travers les processus familiaux du style parental et des pratiques parentales de suivi scolaire, est donc associé à l'ajustement scolaire de l'adolescent. Cependant, il apparaît que ces pratiques fluctuent selon les caractéristiques du jeune et de la période dans le temps au cours de laquelle elles se déroulent. À cet égard, les difficultés présentées par les élèves, l'âge et le genre de ces derniers représentent des paramètres essentiels à considérer dans l'étude de l'engagement parental.

\subsection{Engagement parental et caractéristiques de l'élève}

Une première caractéristique fondamentale qui distingue les adolescents entre eux est le genre. Il est maintenant notoire que les filles et les garçons perçoivent le milieu scolaire différemment et que le lien qui existe entre la famille et l'ajustement s'articule de façon particulière pour chacun des sexes. En effet, plusieurs auteurs soutiennent que les filles sont plus sensibles que les garçons à la relation qu'elles entretiennent avec leurs parents et, qu'en retour, les parents utilisent des pratiques parentales différentes pour un garçon ou pour une fille (Cloutier, 1996; Sartor et Youniss, 2002). Une autre caractéristique propre à l'adolescent, qui concoure à modifier l'engagement du parent, est celle qui renvoie au statut scolaire de l'élève. Ainsi, si l'adolescent est considéré comme présentant des difficultés d'apprentissage ou de comportement, il est probable que le parent interagisse différemment avec lui comparativement à un élève suivant un parcours ordinaire. De fait, une étude de Deslandes, Leclerc et Doré-Côté (2001) montre que les adolescents en classes spéciales ou en cheminements particuliers, comparativement à ceux qui sont en classes ordinaires, sont moins supervisés et sont moins encouragés à faire preuve d'autonomie par leurs parents. En outre, ces élèves reçoivent moins de soutien affectif dans leurs apprentissages et leurs parents communiquent plus fréquemment avec l'enseignant et l'école. Finalement, une étude de Marcotte (2004) révèle que le degré général d'engagement parental diminue alors que l'encouragement à l'autonomie augmente à mesure que les adolescents avancent en âge et ce, peu importe le statut scolaire de l'élève. L'âge du jeune serait donc un élément supplémentaire à considérer, d'autant plus que l'adolescence constitue une période transitionnelle riche en changements.

En résumé, les connaissances disponibles sur la famille et les adolescents montrent que l'engagement parental joue un rôle déterminant dans l'ajustement scolaire des jeunes (Deslandes, 1996). Toutefois, l'investissement dont font preuve les parents est nuancé par les caractéristiques propres aux jeunes. Parmi celles-ci, le genre, les difficultés de l'élève et son âge interviennent dans l'engagement des parents. Or, on déplore une absence d'études traitant précisément de l'évolution dans le temps de l'engagement parental à partir d'un examen combiné des difficultés et du genre de l'élève. C'est précisément sur ce plan que notre étude ${ }^{1}$ veut contribuer à développer les connaissances en répondant à deux questions :

Comment se traduit l'évolution de l'engagement parental selon que l'élève présente des difficultés de comportement ou qu'il est en classe ordinaire?

1 Cet article a été rendu possible grâce au financement du Conseil de recherche en sciences humaines du Canada (CRSH), du Conseil québécois pour la recherche sociale (CQRS) et du Fonds pour la formation de chercheurs et l'aide à la recherche (FCAR) (programme de bourse doctorale). 
Comment ces profils d'engagement parental évoluent-ils en fonction du genre de l'élève?

Les réponses à ces questions sont pertinentes pour les intervenants scolaires qui œuvrent auprès des jeunes et de leurs familles afin de déterminer quels élèves sont susceptibles d'avoir des parents engagés et à quel moment du cheminement scolaire cet engagement est-il plus significatif pour eux dans le but de créer des alliances collaboratives stratégiques et efficaces.

\section{Méthode}

L'ensemble des données de la présente étude est issu d'une recherche longitudinale de huit ans portant sur les facteurs explicatifs de l'abandon scolaire (Fortin, Royer, Potvin et Marcotte, 2001). Cette vaste étude utilise un schème à mesures répétées au sein duquel les sujets ont été interrogés à chaque année pendant huit ans à partir de leur entrée au secondaire. Dans le présent article, nous nous intéressons à deux temps précis de cette étude, soit l'entrée au secondaire (temps 1) et deux ans plus tard (temps 2), ce qui correspond, dans un parcours ordinaire, à la première et troisième secondaire ${ }^{2}$.

\subsection{Participants}

L'échantillon est constitué de 300 élèves dont 150 suivent un cheminement ordinaire et 150 sont considérés comme étant en difficulté. Les élèves en difficulté ont été sélectionnés parce qu'ils affichaient les caractéristiques suivantes : l'élève s'est vu attribué un code d' « élèves handicapés ou en difficulté d'adaptation ou d'apprentissage» (EHDAA) par le ministère de l'Éducation ou le niveau scolaire de l'élève est catégorisé «autre» au temps 1, ce qui signifie qu'il poursuit un cheminement particulier ou est placé dans une classe spéciale. Dans tous les cas de jeunes en difficulté, l'évaluation de l'enseignant des problèmes de comportements extériorisés (Gresham, 1988), place le jeune à au moins un écart-type au-dessus de la moyenne des jeunes de son âge. Pour chacun des élèves en difficulté, un élève ordinaire lui a été apparié en fonction du sexe, de la région de provenance, du revenu familial et de la structure familiale. Les filles composent $30 \%(\mathrm{~N}=90)$ de l'échantillon et les garçons, $70 \%(\mathrm{~N}=210)$. On ne déplore aucune perte de sujet entre les deux temps de mesure.

\subsection{Mesures}

\subsubsection{Style parental ou le Parental Style}

Ce questionnaire (Steinberg et al., 1992), complété par le jeune, comporte trois échelles qui renvoient à la sensibilité parentale ${ }^{3}$ ( 9 items), l'encouragement à l'autonomie ( 9 items) et

2 Les âges de 13 et 15 ans ont été considérés étant donné les changements majeurs qui interviennent pendant ces années en ce qui a trait au développement de l'adolescent (Cloutier, 1996).

3 Nous avons préféré l'emploi du terme sensibilité parentale à celui d'engagement parental pour éviter les confusions possibles entre cette sous-échelle du style parental et le score d'engagement global également utilisé dans cette étude. La sous-échelle de la sensibilité parentale renvoie au sentiment que le parent est disponible et à l'écoute du jeune lorsque celui-ci a des problèmes personnels alors que le score global renvoie à l'addition des différentes dimensions du style parental et des pratiques parentales de suivi scolaire. 
l'encadrement parental ( 8 items). Les indices de consistance interne de ces échelles de type Likert s'avèrent tout à fait satisfaisants et la validité de construit et de contenu a été démontrée lors de recherches québécoises auprès d'adolescents (Deslandes, Bertrand, Royer et Turcotte, 1995).

\subsubsection{Pratiques parentales de suivi scolaire}

Traduit et validé au Québec par Deslandes et al. (1995), ce questionnaire (Epstein, 1992), complété par le jeune, évalue la participation parentale au suivi scolaire. Il est composé de 20 énoncés mesurant la participation des parents à partir d'activités à la maison ou à l'école. Ces énoncés sont répartis en quatre sous-échelles: interaction scolaire (4 items, comme: «Mon parent me questionne sur l'école.»), communication parents-école (3 items, comme: "Mon parent assiste aux réunions de l'école.»), soutien affectif ( 6 items, comme: «Mon parent me félicite pour mes réalisations. ») et communication parents-enseignants (4 items, comme: «Mon parent parle avec tous mes enseignants.»). Les réponses des élèves sont cotées sur une échelle de type Likert à quatre points (jamais, une fois, quelquefois et souvent). La consistance interne de la version québécoise se compare à celle de la version américaine et les indices varient de 0,65 à 0,76 . Pour les besoins particuliers de cette étude, un score d'engagement global est calculé à partir des dimensions du style et des pratiques parentales au suivi scolaire.

\subsection{Procédure}

Les élèves ont été rejoints par l'entremise de l'enseignant qui a accepté de participer. Ensuite, les parents ou les tuteurs légaux ont été sollicités afin d'obtenir leur consentement écrit et de leur assurer le traitement confidentiel des données. Les élèves ont été informés des contacts ultérieurs (annuellement) et de la possibilité de mettre un terme à l'étude en tout temps. Les questionnaires des jeunes et des enseignants ont été complétés en groupes-classes à un moment précis de l'année scolaire.

\section{Résultats}

Les objectifs de cette étude sont, d'une part, d'étudier l'évolution de l'engagement parental en fonction du groupe d'élèves (difficulté ou ordinaire) et, d'autre part, de connaître l'effet du genre sur ces profils évolutifs au fil du temps. Des comparaisons de moyennes préliminaires, effectuées à l'aide du test-t, ont démontré des différences significatives entre les groupes quant aux différentes mesures d'engagement. Au premier temps de mesure, les élèves ordinaires perçoivent leurs parents comme étant significativement plus sensibles $[t(300)=2,67 ; p<0,01]$, plus encadrants $[t(300)=4,47 ; p<0,001]$ et plus disposés à les encourager $[t(300)=2,60 ; p<$ $0,01]$ vers l'autonomie que les élèves en difficulté de comportement. Du côté des pratiques de suivi scolaire, seule la mesure de soutien affectif différencie les deux groupes. En effet, les élèves ordinaires reçoivent significativement plus de soutien dans leur suivi scolaire $[t(300)=4,37 ; p<$ $0,001]$. Finalement, le score global d'engagement parental perçu des élèves est significativement plus élevé chez les élèves ordinaires comparativement à leurs camarades présentant des difficultés $[t(300)=3,02 ; p<0,01]$. Au deuxième temps de mesure, les élèves ordinaires perçoivent significativement plus d'encadrement $[t(300)=4,38 ; p<0,001]$, d'encouragement à l'autonomie 
$[\mathrm{t}(300)=3,08 ; \mathrm{p}<0,01]$ et de soutien affectif dans leur parcours scolaire $[t(300)=3,13 ; \mathrm{p}<0,01]$ de la part de leurs parents comparativement à leurs comparses en difficulté. Le tableau 1 présente en détail les comparaisons de moyennes pour les différentes mesures.

Tableau 1

Comparaison de moyennes aux mesures d'engagement parental en fonction du groupe d'élèves aux deux temps de mesure

\begin{tabular}{|c|c|c|c|}
\hline & Élèves ordinaires & $\begin{array}{l}\text { Élèves en difficulté } \\
\text { de comportement }\end{array}$ & \\
\hline Mesures d'engagement & M (é.t.) & M (é.t.) & $t$ \\
\hline \multicolumn{4}{|l|}{ Temps 1} \\
\hline \multicolumn{4}{|l|}{ Style parental } \\
\hline Sensibilité parentale & $3,42(0,50)$ & $3,26(0,56)$ & $2,67^{* * *}$ \\
\hline Encadrement parental & $2,36(0,45)$ & $2,12(0,46)$ & $4,47 * * *$ \\
\hline Encouragement à l'autonomie & $2,38(0,56)$ & $2,21(0,60)$ & $2,60 * *$ \\
\hline \multicolumn{4}{|l|}{ Pratiques de suivi scolaire } \\
\hline Interaction scolaire & $3,28(0,63)$ & $3,19(0,57)$ & 1,22 n.s. \\
\hline Communication parents-école & $2,24(0,64)$ & $2,22(0,71)$ & 0,15 n.s. \\
\hline Soutien affectif & $3,09(0,65)$ & $2,78(0,61)$ & $4,37^{* * *}$ \\
\hline Communication parents-enseignants & $2,07(0,69)$ & $2,15(0,70)$ & $-1,05$ \\
\hline \multicolumn{4}{|l|}{ Mesure globale ${ }^{4}$} \\
\hline Score total engagement & $21,23(3,06)$ & $20,20(2,85)$ & $3,02 * *$ \\
\hline \multicolumn{4}{|l|}{ Temps 2} \\
\hline \multicolumn{4}{|l|}{ Style parental } \\
\hline Sensibilité parentale & $3,18(0,48)$ & $3,11(0,44)$ & 1,38 n.s. \\
\hline Encadrement parental & $2,36(0,45)$ & $2,12(0,48)$ & $4,38^{* * *}$ \\
\hline Encouragement à l'autonomie & $2,69(0,63)$ & $2,46(0,66)$ & $3,08^{* *}$ \\
\hline \multicolumn{4}{|l|}{ Pratiques de suivi scolaire } \\
\hline Interaction scolaire & $3,07(0,63)$ & $3,00(0,72)$ & 0,91 n.s. \\
\hline Communication parents-école & $1,83(0,59)$ & $1,92(0,72)$ & $-1,18$ n.s. \\
\hline Soutien affectif & $2,79(0,68)$ & $2,19(0,68)$ & $3,13 * *$ \\
\hline Communication parents-enseignants & $1,80(0,63)$ & $1,92(0,67)$ & $-1,62$ n.s. \\
\hline \multicolumn{4}{|l|}{ Mesure globale } \\
\hline Score total engagement & $19,96(2,98)$ & $19,28(3,12)$ & 1,90 n.s. \\
\hline
\end{tabular}

Légende: ${ }^{*} \mathrm{p}<0,05 ; * * \mathrm{p}<0,01 ; * * * \mathrm{p}<0,001$.

Ensuite, à l'aide d'une analyse de variance à mesures répétées, nous avons vérifié l'évolution de l'engagement parental entre les deux temps de mesure en fonction du groupe d'appartenance des élèves et du genre. Les résultats de l'analyse effectuée sur le score global d'engagement montre une baisse significative de l'engagement parental entre le temps 1 et le temps $2[F(1,292)=27,04 ; p<0,001]$ et ce, peu importe le groupe d'appartenance de l'élève. Toutefois, les résultats montrent une interaction significative du genre avec la baisse d'engagement parental observée $[\mathrm{F}(2,292)=4,22 ; \mathrm{p}<0,05]$. Les figures 1,2 et 3 , ci-dessous, présentent

4 La mesure globale est calculée à partir des 7 dimensions du style et des pratiques parentales. 
respectivement la variation du score total d'engagement en fonction du groupe d'appartenance, la variation du score total d'engagement en fonction du groupe chez les garçons et finalement, la même variation chez les filles.

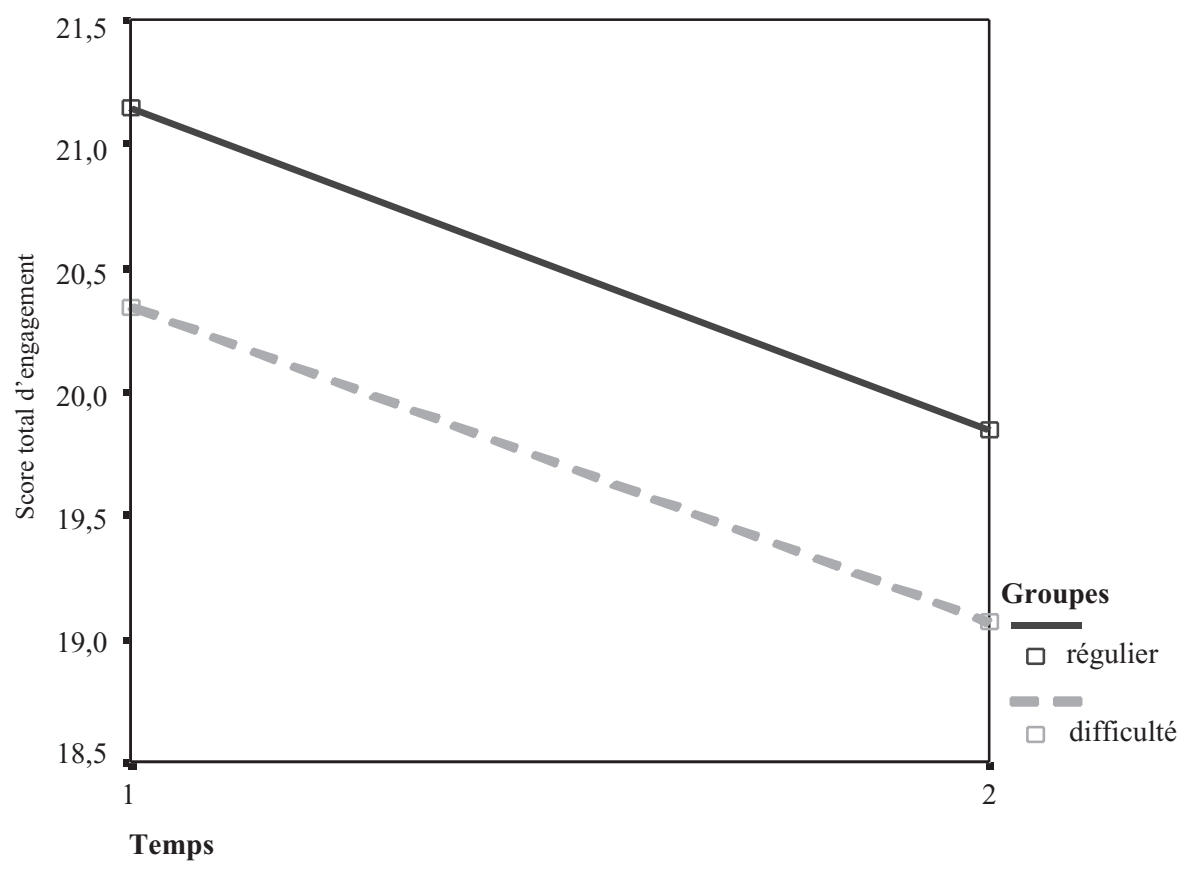

Figure 1 - Variation de l'engagement parental perçu entre les deux temps de mesure selon le groupe d'élèves

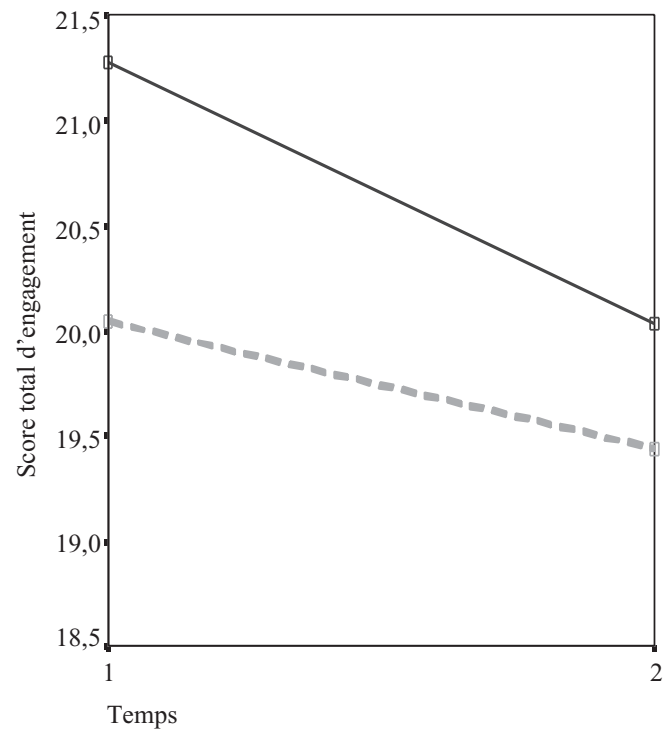

Figure 2 - Variation de l'engagement parental chez les garçons

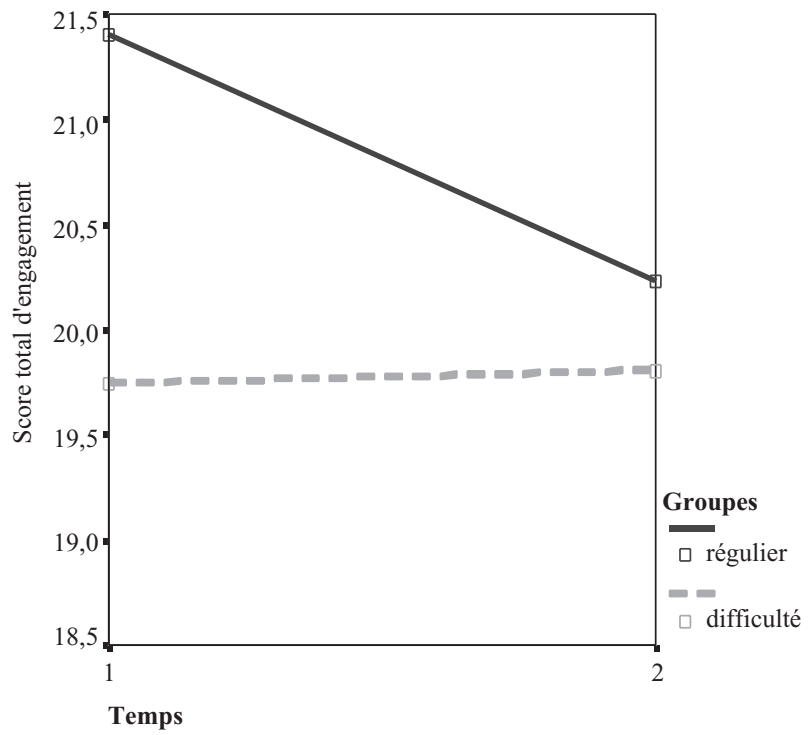

Figure 3 - Variation de l'engagement parental chez les filles

Nous avons effectué, par la suite, des analyses de variance à mesures répétées sur chacune des dimensions de l'engagement parental, c'est-à-dire sur les mesures du style parental et des pratiques parentales de suivi scolaire. Les résultats montrent que les jeunes perçoivent une baisse 
significative de la sensibilité parentale $[\mathrm{F}(1,296)=27,73 ; \mathrm{p}<0,001]$, un maintien dans l'encadrement parental $[\mathrm{F}(1,296)=0,64 ;$ n.s. $]$ et une hausse de l'encouragement à 1 'autonomie $[\mathrm{F}(1,296)=$ 47,54; $\mathrm{p}<0,001]$. Du côté des pratiques parentales de suivi scolaire, les jeunes perçoivent une baisse pour toutes les mesures : l'interaction scolaire entre les parents et les jeunes $[\mathrm{F}(1,296)=$ $26,61 ; \mathrm{p}<0,001]$, les communications parents-école $[\mathrm{F}(1,296)=53,16 ; \mathrm{p}<0,001]$, le soutien affectif $[\mathrm{F}(1,296)=34,84 ; \mathrm{p}<0,001]$ et les communications parents-enseignants $[\mathrm{F}(1,296)=26,00$; $\mathrm{p}<0,001]$. La variation dans le temps de deux mesures comporte des interactions. Tout d'abord, on remarque une interaction significative entre le niveau de la sensibilité parentale, le genre de l'élève et l'appartenance au groupe en difficulté de comportement ou ordinaire $[\mathrm{F}(1,296)=4,56$; $\mathrm{p}<0,05]$. Plus spécifiquement, les analyses selon le genre montrent une hausse de la sensibilité parentale chez les filles en difficulté de telle sorte que ce score est plus élevé chez ces dernières comparativement à leurs pairs féminines suivant un parcours ordinaire lors du deuxième temps de mesure. Chez les garçons, on note une baisse de cette mesure et elle est comparable pour les deux groupes.

L'encadrement parental constitue la deuxième mesure qui présente des interactions en fonction du temps. Ainsi, bien que le score demeure stable au fil du temps pour les jeunes en général, on note une interaction significative entre l'encadrement perçu, le genre et le groupe d'appartenance de l'élève $[F(1,296)=7,34 ; p<0,001]$. L'étude des différences permet de constater que les filles en difficulté de comportement, comparativement aux filles suivant un parcours ordinaire, perçoivent une hausse de l'encadrement parental entre les deux temps de mesure alors que chez les garçons des deux groupes, les scores demeurent relativement stables avec une tendance à la baisse pour les élèves masculins en difficulté de comportement.

\section{Discussion}

Nous pouvons, grâce aux résultats obtenus dans le cadre de cette démarche, identifier trois constats majeurs. D'une part, les différences significatives observées quant à l'engagement global semblent démontrer clairement que les jeunes présentant des difficultés perçoivent une sensibilité moins grande, une supervision moins serrée et moins d'encouragement à l'autonomie de la part de leurs parents, en plus d'obtenir moins d'encouragement relativement à leur suivi scolaire. Autrement dit, les parents des élèves en troubles de comportement semblent moins à l'écoute pour résoudre les problèmes personnels de leurs jeunes (sensibilité), sont moins au courant de leurs allées et venues (encadrement) et encouragent moins l'expression de leur individualité au sein de la famille (autonomie) en plus d'être moins impliqués dans leurs apprentissages scolaires (soutien affectif). Les différences entre les groupes semblent s'estomper entre les deux temps de mesure, mais l'autonomie, l'encadrement et le soutien affectif demeurent moindres chez les jeunes en difficulté. Ces résultats corroborent ceux de Deslandes et al. (2001) qui soulèvent sensiblement les mêmes différences entre les groupes. Plusieurs auteurs mentionnent que les échecs répétitifs et les troubles de comportement amènent les parents à se détacher du processus éducationnel de leur enfant au sein duquel ils se sentent impuissants (Epstein, 2001). D'autres chercheurs posent l'hypothèse que les élèves en difficulté ont une perception erronée de la réalité familiale (Darling et Steinberg, 1993) et des personnes susceptibles de les aider (Bradette, Royer, Marcotte, Potvin et Fortin, 1999). 
D'autre part, les analyses réalisées en fonction du temps, nous permettent de dégager un profil d'engagement global à la baisse, semblable pour les deux groupes. Lorsque cette baisse est observée en fonction des différentes dimensions du style et des pratiques parentales de suivi scolaire, les deux groupes de jeunes perçoivent une diminution significative de l'engagement parental, un maintien du niveau de supervision et une augmentation de l'encouragement à l'autonomie. De leur côté, toutes les pratiques parentales de suivi scolaire sont perçues comme ayant moins d'impact au deuxième temps de mesure. Ce profil évolutif de l'engagement parental a déjà été observé auprès d'un groupe d'élèves ordinaires (Marcotte, 2004) et en difficulté (Deslandes et al., 2001). Il est possible que le jeune, ayant en moyenne 15 ans, perçoit une adaptation saine du parent qui l'encourage davantage à prendre ses propres décisions et à émettre ses opinions tout en continuant de le superviser et en lui accordant une confiance plus grande relativement à son suivi scolaire qui se traduit par une baisse de l'implication perçue sur cet aspect (Marcotte, 2004). Le développement de l'identité et du concept de soi (self-concept), propre à cet âge, peuvent également modifier la perception qu'a le jeune du rôle de ses parents et concourir à l'identification de ce profil évolutif d'engagement parental (Oosterwegel et Oppenheimer, 1983).

Finalement, les analyses à mesures répétées effectuées en fonction du genre de l'élève apportent des nuances importantes. Ainsi, le profil évolutif de l'engagement global identifié pour les groupes diffère sensiblement pour les filles en difficulté de comportement. Ces dernières tendent à percevoir une sensibilité et un encadrement parental plus élevés au deuxième temps de mesure, accompagnés (comme pour les autres) d'une hausse de l'encouragement à l'autonomie et d'une baisse des pratiques parentales de suivi scolaire. Donc, contrairement aux filles qui poursuivent un cheminement ordinaire, les filles présentant des difficultés discernent une supervision accrue - une meilleure connaissance des déplacements et des heures de sorties -, une sensibilité plus grande de leurs parents de même qu'un accueil plus favorable face à leurs opinions et leurs idées à 15 ans que deux ans plus tôt. Du côté des garçons, l'évolution dans le temps du profil d'engagement global ne diffère pas en fonction du groupe d'appartenance. Il est donc permis de penser que les parents de filles en difficulté de comportement, ayant pris conscience des problèmes qu'elles affichent en contexte scolaire, assurent un suivi plus serré et une implication plus grande tout en favorisant leur autonomie, ce qui représenterait un profil adapté à la réalité scolaire de ces dernières. En outre, le fait que ce profil ne soit pas caractéristique des garçons en difficulté de comportement remet au premier plan les différences garçons-filles quant au processus de scolarisation, et ce, au sein même du milieu familial qui représente le premier lieu de socialisation.

Pour conclure, cette étude comporte certaines lacunes dont la principale est de se fier uniquement aux perceptions qu'ont les jeunes de la mobilisation parentale. Les résultats auraient été bonifiés d'une comparaison de la perception des parents et des jeunes du style et des pratiques parentales. Il demeure que cette étude a permis de mettre en relief des constats importants relativement à la perception qu'ont les jeunes de l'implication manifestée par leurs parents à leur égard. Si les élèves en difficulté de comportement identifient leurs parents comme étant moins impliqués que les élèves ordinaires, les deux groupes perçoivent une baisse générale de l'implication à mesure qu'ils vieillissent. Or, ce profil évolutif est différent chez les filles en difficulté de comportement qui constitue le seul sous-groupe à bénéficier d'un encadrement plus serré et d'une sensibilité accrue de la part des parents au fil du temps. Les recherches futures devraient considérer l'interaction genre-difficulté afin de mieux comprendre l'engagement parental à l'adolescence. En somme, les résultats offrent des pistes d'intervention intéressantes pour les personnes 
œuvrant dans les milieux scolaires. Ils révèlent, en effet, que le début de l'adolescence semble un moment clé pour mettre sur pied une collaboration étroite avec les parents puisque les adolescents perçoivent davantage d'engagement de leur part à ce moment. Aussi, les résultats montrent que les filles, particulièrement celles en difficulté, risquent d'être plus touchées par le maintien dans le temps de ces alliances collaboratives.

\section{Références}

Bogenschneider, K. (1997). Parental involvement in adolescent schooling: A proximal process with transcontextual validity. Journal of Marriage and the Family, 59(3), 718-733.

Bradette, S., Royer, É., Marcotte, D., Potvin, P. et Fortin, L. (1999). Stratégies d'adaptation: comparaison entre des adolescents qui présentent des difficultés scolaires et d'autres qui n'en présentent pas. Revue québécoise de psychologie, 20(3), 61-73.

Cloutier, R. (1996). Psychologie de l'adolescence. Boucherville: Gaëtan Morin.

Darling, N. et Steinberg, L. (1993). Parenting styles as context: An integrative model. Child Development, 113(3), 487-496.

Deslandes, R. (1996). Collaboration entre l'école et les familles: influence du style parental et de la participation parentale sur la réussite scolaire au secondaire. Thèse de doctorat en sciences de l'éducation, Université Laval, Québec.

Deslandes, R., Bertrand, R., Royer, É. et Turcotte, D. (1995). Validation d'instruments de mesure du style parental et de la participation parentale dans le suivi scolaire. Mesure et évaluation en éducation, 18(2), 63-79.

Deslandes, R., Leclerc, D. et Doré-Côté, A. (2001). Longitudinal studies of special education and regular students : Autonomy, parental involvement practices and degree of reciprocity in parent-adolescent interactions. Communication présentée à l'Annual meeting of the American Educational Research Association, Seattle, WA, 10-14 avril.

Epstein, J.L. (1992). School and family partnerships. In M. Alkin (dir.), Encyclopedia of educational research (p. 1139-1151). New York, NY: Macmillan.

Epstein, J.L. (2001). School, family and community partnerships. Preparing educators and improving schools. Boulder, CO: Westview Press.

Fortin, L., Royer, É., Potvin, P. et Marcotte, D. (2001). Revue des facteurs de risque et de protection concernant l'adaptation sociale à l'adolescence. Revue internationale de psychologie sociale, 14(2), 93-120.

Gresham, F.M. (1988). Social skills : Conceptual and applied aspects of assessment, traning and social validation. In J.C. Witt, S.E. Elliot et F.M. Gresham (dir.), Handbook of behavior therapy in education (p. 523-546). New York, NY: Plenum Press.

Holmbeck, G.N., Paikoff, R.L. et Brooks-Gunn, J. (1998). Parenting adolescents. In M.H. Bornstein (dir.), Handbook of Parenting (Vol. 1, p. 91-118). Mahwah, NJ: Lawrence Erlbaum.

Marcotte, J. (2004). L'influence de la famille sur l'ajustement scolaire des adolescents. Thèse de doctorat en psychologie, Université Laval, Québec.

Oosterwegel, A. et Oppenheimer, L. (1983). The self-system: Developmental changes between and within self-concepts. Hillsdale, NJ : Lawrence Erlbaum.

Sartor, C.E. et Youniss, J. (2002). The relationship between positive parental involvement and identity achievement during adolescence. Adolescence, 37(146), 221-234.

Steinberg, L. (2000). Adolescent development. Annual Review of Psychology, 52, 83-110.

Steinberg, L., Lamborn, S.D., Dornbusch, S.M. et Darling, N. (1992). Impact of parenting practices on adolescent adjustment across varied ecological niches. Journal of Research on Adolescence, 1(1), 19-36.

Tap, P. et Oubrayrie, N. (2002). Dynamique transactionnelle et relations de pouvoir entre adolescents et parents: analyse critique de l'empowerment. Document interne. Toulouse: Université Toulouse II Le Mirail.

Wallis, J.R. et Barrett, P.M. (1998). Adolescent adjustment and the transition to high school. Journal of Child and Family Study, 7(1), 43-58. 